\title{
ANTICOAGULAÇÃO EM GESTANTES COM PRÓTESE VALVAR MECÂNICA
}

\author{
Anticoagulation in pregnant women with valve mechanical prosthesis \\ Anticoagulación en gestantes con prótesis valvular mecánica
}

José Lopes Cavalcante Júnior ${ }^{1 *}$, Marlúcia do Nascimento Nobre ${ }^{1}$, Rovanda Guimarães Sena ${ }^{1}$, José Wilson de Souza Cavalcante ${ }^{1}$, Rafaelly Taketomi de Magalhães ${ }^{1}$, Francisco Marcelo Saraiva Luna ${ }^{1}$

\section{RESUMO}

Objetivos: Discutir a estratificação do risco materno ao tromboembolismo, as características dos anticoagulantes indicados para a sua prevenção e as estratégias terapêuticas em grávidas com prótese valvar mecânica nos diferentes momentos da gestação. Métodos: Este artigo é uma revisão da literatura sobre anticoagulação em gestantes com prótese valvar mecânica, por meio de busca e análise de dados dos trabalhos científicos disponíveis. Resultados e Discussões: O estado de hipercoagulabilidade, próprio da gravidez, potencializa o risco de tromboembolismo em gestantes com próteses mecânicas. Desse modo, a anticoagulação não deve ser interrompida durante a gestação e puerpério. Entretanto, várias complicações relacionam-se à terapia anticoagulante, como hemorragias e abortamento. Há também o risco de malformações fetais causadas pelos efeitos teratogênicos da varfarina quando utilizada no primeiro trimestre. Apesar deste efeito temível, é considerada o anticoagulante mais eficaz na proteção contra tromboembolismo. As heparinas são alternativas possíveis, porém menos eficazes. Considerações finais: A maior morbimortalidade nessas pacientes torna necessário cuidados especiais, que vão desde a escolha do tipo de prótese valvar nas mulheres ainda em idade fértil, até o manejo clínico durante o ciclo gravídico-pueperal.

Palavras-chave: Gestação; Valva mecânica; Anticoagulação.

\begin{abstract}
Objectives: To discuss the stratification of maternal risk to thromboembolism, the characteristics of the anticoagulants indicated for its prevention and the therapeutic strategies in pregnant women with mechanical valve prosthesis at different moments of gestation. Methods: This article is a review of the literature on anticoagulation in pregnant women with mechanical valve prosthesis, through the search and analysis of available scientific data. Results and Discussion: The state of hypercoagulability, characteristic of pregnancy, potentiates the risk of thromboembolism in pregnant women with mechanical prostheses. Thus, anticoagulation should not be discontinued during gestation and puerperium. However, several complications are related to anticoagulant therapy, such as bleeding and abortion. There is also the risk of fetal malformations caused by the teratogenic effects of warfarin when used in the first trimester. Despite this fearsome effect, it is considered the most effective anticoagulant in the protection against thromboembolism. Heparins are possible alternatives, but less effective. Final considerations: The higher morbidity and mortality in these patients makes special care necessary, ranging from the choice of type of valve prosthesis in women of childbearing age, to clinical management during the pregnancy-pituitary cycle.
\end{abstract}

Keywords: Gestation; Mechanical valves; Anticoagulation

${ }^{1}$ Universidade Federal do Amazonas (UFAM). Hospital Universitário Francisca Mendes (HUFM). ManausAM. E-mail: jlcavalcantejr@gmail.com 


\section{RESUMEN}

Objetivos: Discutir la estratificación del riesgo materno al tromboembolismo, las características de los anticoagulantes indicados para su prevención y las estrategias terapéuticas en embarazadas con prótesis valvular mecánica en los diferentes momentos de la gestación. Métodos: Este artículo es una revisión de la literatura sobre anticoagulación en gestantes con prótesis valvular mecánica, por medio de búsqueda y análisis de datos de los trabajos científicos disponibles. Resultados y Discusiones: El estado de hipercoagulabilidad, propio del embarazo, potencializa el riesgo de tromboembolismo en gestantes con prótesis mecánicas. De este modo, la anticoagulación no debe ser interrumpida durante la gestación y el puerperio. Sin embargo, varias complicaciones se relacionan con la terapia anticoagulante, como hemorragias y abortos. También existe el riesgo de malformaciones fetales causadas por los efectos teratogénicos de la warfarina cuando se utiliza en el primer trimestre. A pesar de este efecto temible, se considera el anticoagulante más eficaz en la protección contra tromboembolismo. Las heparinas son alternativas posibles, pero menos eficaces. Consideraciones finales: La mayor morbimortalidad en esas pacientes requiere de cuidados especiales, que van desde la elección del tipo de prótesis valvular en las mujeres en edad fértil, hasta el manejo clínico durante el ciclo gravídico-pueperal.

Palabras clave: Embarazo; Válvula mecánica; Anticoagulación.

\section{INTRODUÇÃO}

As diversas alterações fisiológicas que ocorrem no organismo materno durante a gestação conferem um estado de hipercoagulabilidade. As principais modificações responsáveis por essa condição são o aumento da atividade plaquetária, maior produção de fatores de coagulação e menor ação do sistema fibrinolítico. As próteses valvares mecânicas por sua vez, possuem características trombogênicas intrínsecas importantes que, somadas as alterações pró-trombóticas inerentes da gestação, tornam essas pacientes bastante susceptíveis ao desenvolvimento de fenômenos tromboembólicos graves e por vezes fatais. Os riscos são estimados anualmente, independente do ritmo cardíaco, em 12\% para as próteses na posição aórtica e $22 \%$ na posição mitral, na ausência de anticoagulação. A incidência de trombogênese na posição aórtica é menor em razão da maior pressão do fluxo sanguíneo sobre a superfície valvar, reduzindo o depósito de fibrina. (TARASOUTCHI F, et al., 2011).

As gestantes portadoras de próteses valvares mecânicas também apresentam riscos relacionados aos efeitos adversos da anticoagulação durante o período gravídico-puerperal, mas que ao mesmo tempo é fundamental para a viabilidade da gestação. A trombose de prótese valvar e as perdas fetais relacionam-se a uma anticoagulação inadequada. Portanto, o sucesso da gravidez depende de uma rigorosa anticoagulação, visando simultaneamente à eficácia antitrombótica materna e à proteção fetal (ELKAYAM e BITAR, 2005). A varfarina é a droga anticoagulante mais utilizada nessas pacientes, apresentando os melhores resultados em relação à mortalidade materna quando comparada aos outros anticoagulantes disponíveis (WALFISCH e KOREN, 2010). Por outro lado, as heparinas podem ser usadas como opção, sobretudo entre a $6^{\underline{a}}$ e $12^{\underline{a}}$ semanas de gestação, minimizando a exposição fetal aos efeitos teratogênicos da varfarina. (STARLING, et al., 2012).

Segundo o registro de gravidez e doença cardíaca da Sociedade Europeia de Cardiologia, as mulheres portadoras de prótese valvar mecânica tem somente $58 \%$ de probabilidade de gravidez sem complicações. (HAGEN, et al., 2015). Dessa maneira, é fundamental compreender os principais aspectos no tratamento e seguimento de grávidas com prótese valvar mecânica, enfatizando para a paciente e seus familiares sobre a importância da manutenção e acompanhamento do tratamento durante toda a gravidez e puerpério. É necessário ter conhecimento dos problemas relacionados à gestação para tomar as decisões mais apropriadas, que vão desde a escolha das próteses nas mulheres em idade fértil até o manejo clínico de situações específicas durante a gravidez e o parto (BORN, et al., 1992). 


\section{PRÓTESES VALVARES E GESTAÇÃO}

Não é raro em sua prática clínica, o médico cardiologista atender mulheres em idade fértil que são portadoras de valvopatias com indicação de troca valvar como terapia definitiva. Dois grupos de próteses artificiais estão disponíveis nesse contexto: mecânicas e biológicas. É fundamental conhecer as principais características de cada um desses grupos, uma vez que de acordo com o tipo de prótese escolhida, poderá ter grande impacto na morbimortalidade em caso de uma eventual gestação. As próteses artificiais diferem entre si pela trombogenicidade e durabilidade (HAGEN, et al., 2015).

As próteses mecânicas são naturalmente trombogênicas, elevando acentuadamente os riscos de complicações tromboembólicas. Tais eventos em gestantes com próteses mecânicas têm incidência entre 10$15 \%$ (BORN, et al., 1992). Aproximadamente $2 / 3$ dessas pacientes apresentam trombose valvar, levando à morte em $40 \%$ dos casos (LEE, et al., 1994). A presença de fibrilação atrial, próteses de gerações antigas, eventos prévios e posição mitral, aumentam os riscos (HAGEN, et al., 2015). Dessa maneira, a terapia anticoagulante plena no período gravídico-puerperal é fundamental para prevenção dessas complicações. Entretanto, pode ser acompanhada de efeitos indesejáveis tanto para mãe quanto para o feto. Já as próteses biológicas não são trombogênicas e não necessitam de anticoagulação permanente (HUGHES e LIP, 2007).

Um importante aspecto limita o uso das biopróteses em pacientes jovens, a durabilidade. Sua meia-vida é significativamente inferior quando comparada as mecânicas. Cerca de $30 \%$ das biopróteses necessitam de troca em um período médio de 10 anos e existem algumas evidências de que fatores como idade jovem e gravidez possam acelerar a degeneração valvar e antecipar sua substituição (ELKAYAM e KAHN, 1998). Uma nova retroca pode estar associada a maior morbimortalidade. Por outro lado, as próteses mecânicas duram de 25 à 40 anos ou são ilimitadas.

Diante das características dos diferentes tipos de próteses valvares já discutidas, existem divergências na literatura em relação à melhor opção para as pacientes com o desejo de engravidar (HUGHES e LIP, 2007). É fundamental que todas as informações e dúvidas sejam esclarecidas afim de que a escolha seja individualizada e embasada nos dados presentes na literatura.

\section{ANTICOAGULAÇÃO EM GESTANTES COM PRÓTESES VALVARES MECÂNICAS}

A trombose da prótese é a complicação mais temida nessas pacientes, sendo a principal causa de óbito. Deve ser suspeitada em pacientes que evoluem com dispneia de início súbito, edema agudo de pulmão, síncope e sinais de baixo débito cardíaco. Na suspeita desta complicação, deve ser realizado imediatamente um ecocardiograma transtorácico, seguido geralmente do ecocardiograma transesofágico que possui maior sensibilidade para o diagnóstico. De maneira geral, pacientes com classe funcional III ou IV da NYHA, trombos maiores que $10 \mathrm{~mm}$ e trombose de prótese mitral ou aórtica, a primeira opção é pelo tratamento cirúrgico. A cirurgia associa-se com alta mortalidade perioperatória, entre 5 e 18\%, estreitamente associada à classe funcional, sendo este o principal preditor. Nos demais casos (NYHA I ou II, trombos à direita, ou menores que $10 \mathrm{~mm}$ de diâmetro) o uso de fibrinolíticos deve ser considerado, podendo ocorrer complicações como embolização sistêmica, sangramentos e trombose residual, necessitando de intervenção cirúrgica de urgência. Em comparação à trombólise, a cirurgia apresenta as maiores taxas de sucesso, 81\% vs. 70,9\% (HUANG, et al., 2013).

O elevado risco de trombose de prótese e embolia sistêmica, torna necessária a manutenção da anticoagulação nessas pacientes. É consenso que a terapia anticoagulante deva ser mantida durante toda a gestação, com um acompanhamento semanal para avaliar se os alvos terapêuticos, que garantem uma anticoagulação plena, estão sendo atingidos. Ao mesmo tempo procura-se evitar os efeitos adversos de uma anticoagulação excessiva. Neste cenário os estudos demonstraram ser possível o uso de três drogas: antagonistas da vitamina K (varfarina), heparina não fracionada (HNF) e heparina de baixo peso molecular (HBPM) (LEE, et al., 1994). 
- Varfarina: Trata-de de um antagonista da vitamina $K$ que reduz a atividade biológica dos fatores de coagulação dependentes de vitamina K (II, VII, IX e X). É considerada o anticoagulante mais eficaz na prevenção de complicações tromboembólicas em gestantes com próteses valvares mecânicas, entretanto 0 seu uso associa-se a uma série de complicações maternas e fetais (WALFISCH e KOREN, 2010). A molécula de varfarina possui baixo peso molecular com capacidade de atravessar a barreira placentária. É teratogênica, sendo o seu efeito indesejável mais temido a embriopatia varfarínica, que ocorre em média em $6,4 \%$ das gestações e compreende um conjunto de malformações fetais características relacionadas ao uso dos antagonistas da vitamina $\mathrm{K}$ no primeiro trimestre, período mais crítico da embriogênese fetal. As anomalias fetais mais comuns são a hipoplasia nasal e a condrodisplasia punctata das epífises dos ossos longos e das vértebras cervicais e lombares. É importante ressaltar que a embriopatia varfarínica é dose-dependente (STARLING, et al., 2012). Estudos demonstraram que doses inferiores a $5 \mathrm{mg}$ ao dia estão associadas a um risco de malformações de $3 \%$, enquanto doses diárias acima de $5 \mathrm{mg}$ elevam esse risco para $8 \%$. Outras complicações atribuídas ao uso da varfarina são abortamento $(10-30 \%)$, hemorragia intracraniana fetal $(15 \%)$ e hemorragias maternas $(5 \%)$, sobretudo no terceiro trimestre e período puerperal.

- HNF: Liga-se ao complexo trombina-antitrombina potencializando a ação da antitrombina em até 1000 vezes. Não atravessa a barreira placentária e é isenta de efeito teratogênico, podendo ser uma opção ao uso da varfarina no primeiro trimestre, minimizando o risco de embriopatia. Alguns autores consideram o seu uso durante toda a gestação, evitando dessa forma qualquer exposição do feto aos antagonistas da vitamina $\mathrm{K}$. No entanto, o uso prolongado da heparina não fracionada está associado à osteoporose, fraturas patológicas, hemorragias maternas e trombocitopenia (CHAN, et al., 2000). Além disso, estudos retrospectivos demonstraram ser um anticoagulante menos eficaz, relacionando-se a uma maior incidência de complicações tromboembólicas e consequentemente maior mortalidade materna. Usada isoladamente durante toda a gravidez, apresentou maior taxa de trombose valvar $(9,2 \%)$ vs utilização de HNF no $1^{\circ}$ trimestre e restante gravidez com anticoagulante oral (3,9\%) (QUINN, et al., 2009)

- HBPM: É um inibidor do fator Xa, apresentando vantagens sobre a HNF por ter melhor biodisponibilidade, um efeito anticoagulante mais previsível e sustentado, baixo risco de osteoporose e trombocitopenia. Não tem ação teratogênica e seu uso também pode ser considerado nessas pacientes. Entretanto, a sua eficácia e perfil de segurança ainda são controversos, necessitando de ensaios randomizados para confirmação de seus reais efeitos. Estudo retrospectivo demonstrou que o uso da heparina de baixo peso molecular em grávidas, pode estar associado com uma incidência aumentada de complicações tromboembólicas em compação à varfarina (BASUDE, et al., 2012). Porém, esse fato parece estar relacionado ao uso de doses subterapêuticas de heparina de baixo peso molecular, incapazes de atingir um nível adequado do fator anti-Xa que garanta uma anticoagulação eficaz (MCLINTOCK, et al., 2009). Sendo assim, na gestação podem ser necessárias doses crescentes de enoxaparina para que o alvo terapêutico seja alcançado, uma vez que o aumento fisiológico da depuração renal na gravidez e também o ganho progressivo de peso da mãe, tornam necessários ajustes na posologia.

Não existe consenso na literatura em relação ao esquema ideal de anticoagulação. Todos os esquemas disponíveis envolvem riscos para a mãe e feto. São necessários estudos randomizados para estabelecer a verdadeira eficácia e segurança entre os diferentes anticoagulantes. As diretrizes brasileira (TARASOUTCHI F, et al., 2011), europeia (VERA, et al., 2011) e americana (NISHIMURA, et al., 2014), recomendam manter o uso de varfarina no segundo e terceiro trimestre, quando o risco de embriopatia pela exposição à droga é baixo. Ao atingir-se a $36^{\text {a }}$ semana é sugerido substituir a varfarina por HNF ou HBPM, por apresentarem uma meia vida mais curta (AVILA e GRINBERG, 2005). Estas devem ser suspensas respectivamente, 4 e 24 horas antes do trabalho de parto e reintroduzidas juntamente com varfarina algumas horas após o mesmo, de acordo com a hemostasia e o risco de sangramento periparto. Quando o INR alvo for atingido, a heparina poderá então ser interrompida.

A principal discussão é em relação a anticoagulação durante o primeiro trimestre. Sabe-se que a varfarina nesse período aumenta o risco de malformação fetal. Por outro lado, sua substitução por heparina 
eleva o risco de tromboembolismo. Em geral, recomenda-se para mulheres que utilizam doses de varfarina inferiores a $5 \mathrm{mg}$ ao dia a sua manutenção, devendo a família ser informada do baixo risco de embriopatia nessa dosagem. Já quando doses acima de $5 \mathrm{mg}$ ao dia são requeridas para atingir uma anticoagulação eficaz, é prudente antes da $6^{\underline{a}}$ semana a substituição por HNF ou HBPM, devendo ser continuadas até a $12^{\mathrm{a}}$ semana de gravidez, quando a varfarina poderá ser novamente reiniciada. Algumas literaturas recomendam o uso de heparina no primeiro trimestre independente da dose de varfarina (VERA, et al., 2011).

A American Heart Association/American College of Cardiology sugere a associação de AAS em baixas doses (75 - $100 \mathrm{mg} /$ dia) junto ao esquema de anticoagulação, para otimizar o efeito antitrombótico, reduzindo a mortalidade, o risco de acidente vascular encefálico e a trombose de prótese (CLASP, 1994). Já a European Society of Cardiology contraindica o uso concomitante de AAS por não haverem estudos controlados demonstrando seu benefício e segurança. A Sociedade Brasileira de Cardiologia considera a prescrição conjunta de AAS para aquelas pacientes consideradas de alto risco (Fluxograma 1).

Fluxograma 1: Esquema de anticoagulação em gestantes com prótese valvar mecânica.

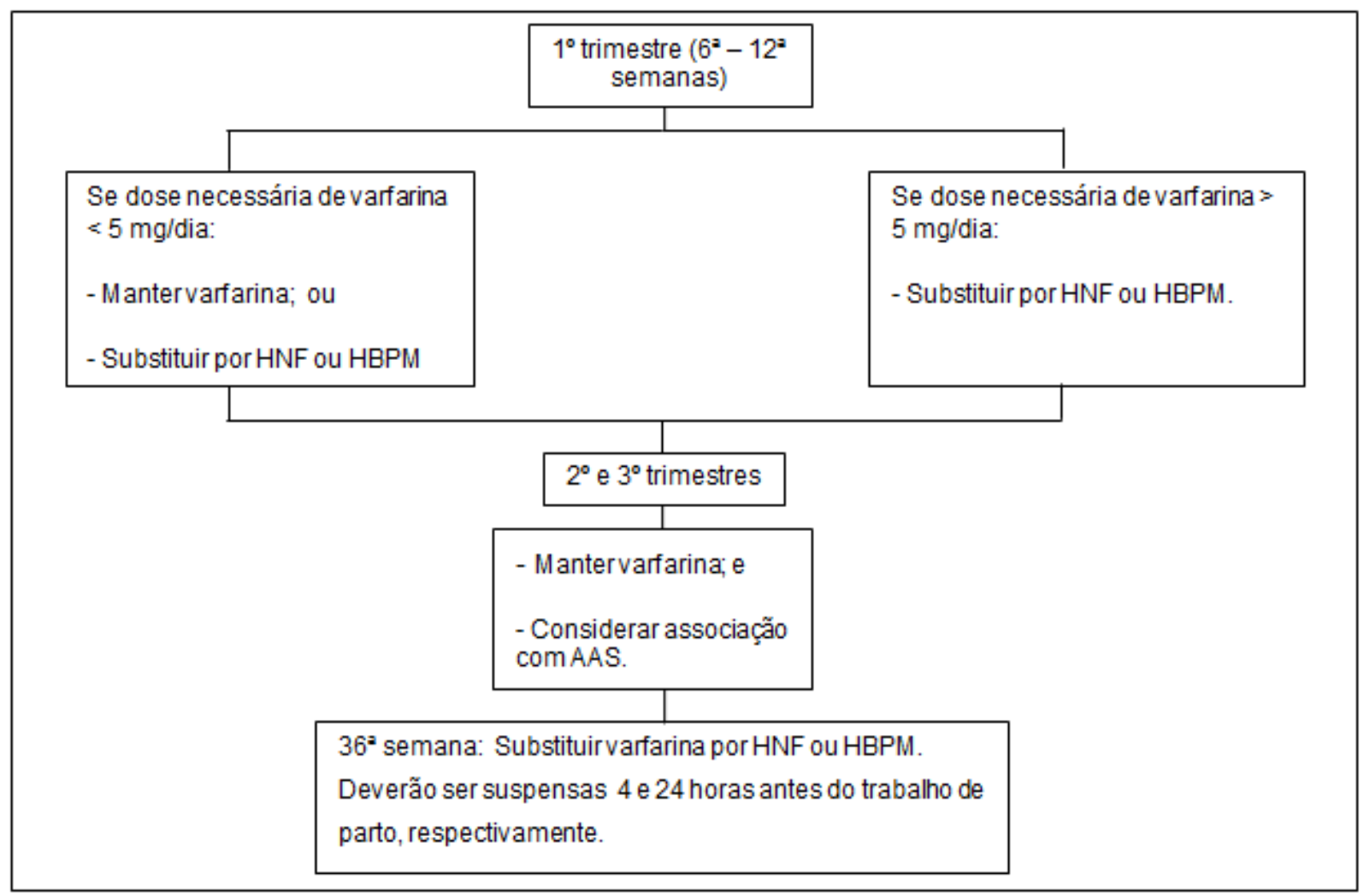

Fonte: Baseado no estudo de Nishimura, et al., 2014 AHA/ACC Valvular Heart Disease Guideline

Outra opção de anticoagulação proposta por alguns autores é a de usar durante toda a gestação, heparina não fracionada ou heparina de baixo peso molecular, mesmo no segundo e terceiro trimestres. Entretanto, trata-se de uma esquema difícil e de baixa adesão, em virtude de dificuldades em acesso a estes medicamentos, a via de administração parenteral e os efeitos adversos com o uso prolongado. Além disso, estudos observacionais demonstraram maior risco de tromboembolismo (AVILA e GRINBERG, 2005).

O uso dos novos anticoagulantes (inibidores diretos da trombina e inibidores orais do Fator Xa) é formalmente contraindicado em pacientes com prótese valvar mecânica (TARASOUTCHI F, et al., 2017). 


\section{CONTROLE DA ANTICOAGULAÇÃO}

Deve ser rigoroso e realizado preferencialmente a cada semana. No caso da varfarina, esse acompanhamento é feito através do INR. Para as próteses mecânicas em posição mitral, independente do ritmo cardíaco, deve-se atingir um INR entre 2,5 - 3,5. Próteses mecânicas em posição aórtica, o alvo terapêutico varia com o ritmo cardíaco: entre 2 - 3 se ritmo sinusal e 2,5 - 3,5 se fibrilação atrial estiver presente (TARASOUTCHI F, et al., 2017).

Com HNF é necessário determinar o tempo de tromboplastina parcial ativado (TTPa), medido $4-6$ horas após a administração do medicamento e com alvo de pelo menos duas vezes o valor de controle. Para HBPM deverá ser realizada a dosagem do fator anti Xa $4-6$ horas após a dose (CHAN, et al., 2000). Existem divergências em relação ao nível ideal que garanta uma anticoagulação eficaz, mas a maioria das literaturas recomendam valores entre $0,8-1,2 \mathrm{Ul} / \mathrm{ml}$, necessitando de ensaios controlados para uma indicação mais fidedigna destes valores (MCLINTOCK, et al., 2009).

\section{CONSIDERAÇÕES FINAIS}

As gestantes com próteses valvares mecânicas possuem um elevado risco de complicações tromboembólicas, sendo necessária a manutenção da anticoagulação durante todo o período de gravidez e puerpério. A varfarina é o anticoagulante mais eficaz nessas pacientes, entretanto é teratogênica no primeiro trimestre, sobretudo quando utilizada em doses superiores a $5 \mathrm{mg} / \mathrm{dia}$. As alternativas são HNF ou HBPM que reduzem os riscos de embriopatia, porém as custas de maior chance de tromboembolismo. Deve ser feito um controle rigoroso da anticoagulação pelas dosagens do INR, TTPa e fator anti-Xa, medidos semanalmente.

\section{REFERÊNCIAS}

1. AVILA WS, GRINBERG M. Anticoagulation, pregnancy and cardiopathy. A triad, three dominions and five moments. Arq Bras Cardiol. 2005;84(1):44-8.

2. BASUDE S, et al. Low-molecular-weight heparin or warfarin for anticoagulation in pregnant women with mechanical heart valves: what are the risks? A retrospective observational study. BJOG 2012; 119(8): 1008-13; discussion 12-13.

3. BORN D, et al. Pregnancy in patients with prosthetic heart valves: The effect of anticoagulation on mother, fetus and neonate. Am Heart J 1992; 124: 413-417

4. CHAN, et al. Anticoagulation of pregnant women with mechanical heart valves: a systematic review of the literature. Arch Intern Med. 2000;160(2):191-6.

5. CLASP: a randomised trial of low-dose aspirin for the prevention and treatment of pre-eclampsia among 9364 pregnant women: CLASP (Collaborative Low-dose Aspirin Study in Pregnancy) Collaborative Group. Lancet 1994; 343:619-629.

6. ELKAYAM U, BITAR F. Valvular heart disease and pregnancy: part II: prosthetic valves. J Am Coll Cardiol. 2005;46(3):403-10.

7. ELKAYAM U, KHAN SS. Pregnancy in the patient with artificial heart valve. In: Elkayam U, Gleicher N, eds. Cardiac Problems in Pregnancy. 3rd ed. New York: Wiley-Liss, 1998: 61-78.

8. ESC Guidelines on the management of cardiovascular diseases during pregnancy European Heart Journal (2011) 32, 3147-3197

9. HUANG, et al. Treatment of obstructive thrombosed prosthetic heart valve. J Am Coll Cardiol. 2013;62(19):1731-6

10. HUGHES M, LIP GY. Risk factors for anticoagulation-related bleeding complications in patients with atrial fibrillation: a systematic review. QJM. 2007;100(10):599-607

11. LEE CN, et al. Pregnancy following cardiac prosthetic valve replacement. Obstet Gynecol 1994; 83: 353-356

12. MCLINTOCK C, MCCOWAN ME. Maternal complications and pregnancy outcome in women with mechanical prosthetic heart valves treated with enoxaparin. Maternal medicine. 2009; 1585-1592.

13. NISHIMURA, et al. 2014 AHA/ACC Valvular Heart Disease Guideline

14. QUINN J, et al. Use of high intensity adjusted dose low molecular weight heparin in women with mechanical heart valves during pregnancy: a single-center experience. Haematologica 2009; 94(11): 1608-1612.

15. STARLING LD, et al. Fetal warfarin syndrome. BMJ case reports; Nov 1; 2012.

16. TARASOUTCHI F, et al. Diretriz Brasileira de Valvopatias - SBC 2011 / I Diretriz Interamericana de Valvopatias - SIAC 2011. Arq Bras Cardiol 2011; $97(5$ supl. 1): 1-67

17. TARASOUTCHI F, et al. Atualização das Diretrizes Brasileiras de Valvopatias: Abordagem das Lesões Anatomicamente Importantes. Arq Bras Cardiol 2017; 109(6Supl.2):1-34

18. TEDOLDI CL, et al. Sociedade Brasileira de Cardiologia. Diretriz da Sociedade Brasileira de Cardiologia para Gravidez na Mulher Portadora de Cardiopatia. Arq Bras Cardiol.2009;93(6 supl.1):e110-e178

19. VAN HAGEN IM, et al. Pregnancy in Women with a Mechanical Heart Valve: Data of the European Society of Cardiology Registry of Pregnancy and Cardiac disease (ROPAC). Circulation 2015. Jul 14;132(2):132-142.

20. WALFISCH A, KOREN G. The "warfarin window" in pregnancy: the importance of half-life. J Obstet Gynaecol Can. 2010 Oct; $32(10)$ : 988-989. 